\title{
Lipid-Induced Endoplasmic Reticulum Stress Impairs Selective Autophagy at the Step of Autophagosome-Lysosome Fusion in Hepatocytes
}

\author{
Koichiro Miyagawa, ${ }^{*}$ Shinji Oe, ${ }^{*}$ Yuichi Honma, ${ }^{*}$ Hiroto Izumi, ${ }^{\dagger}$ Ryoko Baba, ${ }^{\ddagger}$ and Masaru Harada*
}

From the Third Department of Internal Medicine,* School of Medicine, University of Occupational and Environmental Health, Kitakyushu; and the Departments of Occupational Pneumology ${ }^{\dagger}$ and Anatomy, ${ }^{\ddagger}$ School of Medicine, University of Occupational and Environmental Health, Kitakyushu, Japan

\author{
Accepted for publication \\ March 4, 2016. \\ Address correspondence to \\ Koichiro Miyagawa, M.D., or \\ Masaru Harada, M.D., Ph.D., \\ Third Department of Internal \\ Medicine, School of Medicine, \\ University of Occupational and \\ Environmental Health, 1-1 \\ Iseigaoka, Yahatanishi-ku, \\ Kitakyushu 807-8555, \\ Japan. E-mail: koichiro@med. \\ uoeh-u.ac.jp or msrharada@ \\ med.uoeh-u.ac.jp.
}

\begin{abstract}
Blockage of hepatic autophagic degradation system occurs in obesity and is associated with the development of nonalcoholic fatty liver disease. However, the mechanism of this blockage remains unclear. We found a high-fat diet induced accumulation of autophagosomes in the mice livers. However, autophagy substrates such as $\mathrm{p} 62$ and ubiquitinated proteins also accumulated in the livers in this model. These findings indicate the possibility that a high-fat diet impairs autophagic flux in the liver. Then, to assess the autophagic flux in more detail, we performed analyses of autophagic flux in cultured hepatocytes exposed to monounsaturated fatty acids (FAs) or saturated FAs (SFAs). SFAs but not monounsaturated FAs suppressed degradation of contents in the autophagosomes. We analyzed each stage of the autophagy pathway (ie, autophagosome formation, autophagosomelysosome fusion, lysosomal degradation) in cultured hepatocytes treated with monounsaturated FAs or SFAs and found that SFAs impaired autophagosome-lysosome fusion. This impairment occurred in an endoplasmic reticulum stress-dependent manner. Moreover, ubiquitin and p62-positive inclusions observed in high-fat diet-fed mice livers and SFA-treated cells were sequestered within autophagosomes. We also found that SFA-induced accumulation of Ser351-phosphorylated p62, which is indispensable for selective autophagy, further increased on administration of a lysosomal proteinase inhibitor. Although lipid-induced endoplasmic reticulum stress interferes with the autophagosomelysosome fusion, selective autophagic sequestration of aggregated proteins is not inhibited. (Am J Pathol 2016, 186: 1861-1873; http://dx.doi.org/10.1016/j.ajpath.2016.03.003)
\end{abstract}

The liver plays a central role in systemic metabolic homeostasis and is associated with the development of metabolic syndrome. ${ }^{1}$ Nonalcoholic fatty liver disease (NAFLD) is the main hepatic manifestation of metabolic syndrome and is closely related to insulin resistance, visceral obesity, and dyslipidemia. ${ }^{2-4}$ The prevalence of NAFLD continues to rise throughout the world, together with the increased prevalence of obesity and diabetes. ${ }^{5}$

The development of hepatic steatosis is associated with elevated concentrations of circulating free fatty acids (FFAs) generated by adipocyte lipolysis. ${ }^{6}$ The marked increase in FFAs in hepatocytes disturbs endoplasmic reticulum (ER) homeostasis, resulting in ER stress, ${ }^{7}$ which is deeply involved in the development of NAFLD. ${ }^{8,9}$ After ER stress, unfolded protein response is activated to restore ER homeostasis. ${ }^{9,10}$ ER-associated protein degradation is one of the ER quality control mechanisms regulated by unfolded protein response. ${ }^{11}$ The aberrant proteins are dislocated to the cytosol and degraded by the ubiquitin-proteasome system, that is, ER-associated protein degradation. ${ }^{11}$ However, an excess of FFAs suppresses the proteasomal degradation pathway. ${ }^{12} \mathrm{We}$ previously reported that proteasome dysfunction activates autophagy as a response to deal with the abnormal accumulation of misfolded proteins. ${ }^{13}$ It was also postulated that autophagy is induced to dispose of misfolded proteins that cannot be degraded by

Supported by JSPS KAKENHI grants 23591000 and 00241175 (M.H.).

Disclosures: Supported in part by Bristol-Myers Squibb grant for research to M.H. 
ER-associated protein degradation. ${ }^{14}$ However, hepatic autophagy was reported to be defective in obesity. ${ }^{15-17}$ Impairment of the ubiquitin-proteasome system and autophagy-lysosome pathway, the main intracellular protein degradation systems, occurs in NAFLD, and this may play an important role in the development of NAFLD and subsequent severe liver diseases, such as cirrhosis and hepatocellular carcinoma. ${ }^{16,18}$

Autophagy is roughly classified into the following three types: macroautophagy, microautophagy, and chaperonemediated autophagy. ${ }^{19,20}$ Macroautophagy (simply referred to as autophagy hereafter) consists of several steps that involve the formation of autophagosomes that subsequently fuse with the lysosomes to become autolysosomes, leading to degradation of the sequestered contents with the use of lysosomal proteases. ${ }^{21}$ Although autophagy has long been recognized as a random cytoplasmic degradation system, autophagy plays a crucial role for intracellular quality control of proteins and various organelles in various tissues, such as neurons and hepatocytes. ${ }^{22,23}$ Thus, suppression of autophagy results in accumulation of abnormal proteins, swollen mitochondria, distended ER, and lipid droplets and induces the formation of intracellular inclusions. ${ }^{13,24-26}$ Blockade of the autophagic degradation system occurs if any stage of the autophagy pathway (ie, the formation of autophagosome, autophagosome-lysosome fusion, lysosomal degradation) is blocked. Although several mechanisms for the impairment of hepatic autophagic flux in obesity were reported, the process has not been fully elucidated. ${ }^{16,27,28}$

In this study, we found that a high-fat diet (HFD) induced prominent accumulation of autophagosomes in the mice livers. However, autophagy substrates such as p62 and ubiquitinated proteins also accumulated in the livers in this model. These findings collectively indicate the possibility that obesity impairs autophagic flux in the liver. Although saturated FAs (SFAs) tend to promote NAFLD, monounsaturated FAs (MUFAs) exert a salutary effect on oxidative stress and ER stress despite that they increase triglyceride accumulation. $^{29,30}$ To elucidate the mechanism of blockage of hepatic autophagic flux in NAFLD, we evaluated the effect of SFAs and MUFAs, respectively, on autophagic flux in cultured hepatocytes. We also evaluated whether ER stress caused by lipid overload modulates hepatic autophagic flux.

\section{Materials and Methods}

\section{Cell Culture}

We used a human hepatoma cell line established from a hepatocellular carcinoma (Huh7) and a highly differentiated immortalized human hepatocyte cell line (OUMS-29). Cells were cultured in humidified atmosphere that contained $5 \%$ $\mathrm{CO}_{2}$ in complete medium composed of Dulbecco's modified Eagle's medium supplemented with $10 \%$ heat-inactivated fetal bovine serum and antibiotics.

\section{Mouse Experiments}

We used wild-type male C57BL/6J mice, 3 months old, purchased from Kyudo Company (Kumamoto, Japan). Male C57BL/6J mice were fed either a control diet $(n=8)$ or a HFD $(n=8)$ for 12 weeks. At the end of the in vivo experiments mice were sacrificed by diethyl ether inhalation, and liver samples were collected. We cut livers into slices and fixed them with $10 \%$ formalin for subsequent hematoxylin and eosin staining, embedded in optimal cutting temperature compound for immunofluorescence staining, or snap-frozen in liquid nitrogen for tissue homogenization and protein analysis. Moreover, we assessed liver sections that were processed for immunofluorescence staining of microtubule-associated protein 1 light chain 3 (LC3; Medical and Biological Laboratories, Nagoya, Japan), rabbit polyclonal antibody against ubiquitin (Dako, Glostrup, Denmark), or mouse monoclonal antibody against p62 (Santa Cruz Biotechnology, Santa Cruz, CA). LC3-positive structures were analyzed in 30 cells of triplicate experiments by a laser scanning confocal microscope (Zeiss 510 Meta with Lasersharp software version 2.5; Carl Zeiss MicroImaging Inc., Jena, Germany). All procedures were reviewed and approved by the Animal Welfare Committee of the University of Occupational and Environmental Health.

\section{Antibodies}

The following antibodies were used: rabbit anti-LC3 (Medical and Biological Laboratories), mouse anti-p62 (Santa Cruz Biotechnology), rabbit anti-phosphorylation of p62 at Ser351 (Medical and Biological Laboratories), rabbit anti-authophagy-related gene 7 (Atg7; Cell Signaling Technology, Danvers, MA), rabbit anti-Beclin 1 (Novus Biologicals, Littleton, CO), mouse anti-ubiquitin (Santa Cruz Biotechnology), rabbit anti-ubiquitin (Dako), rabbit anti-phosphorylation of $\alpha$ subunit of eukaryotic initiation factor 2 (p-eIF2 $\alpha$; Cell Signaling Technology), rabbit antiX-box binding protein-1 (XBP1; Santa Cruz Biotechnology), and mouse anti-actin (Sigma-Aldrich, St. Louis, MO) were purchased from the indicated suppliers. The mouse anti-lysosomal-associated membrane protein 1 (Lamp1) antibody was a kind gift from J.T. August (Johns Hopkins University, Baltimore, MD).

\section{FFA/BSA Complex Solution Preparation}

Palmitoleic acid (C16:1), oleic acid (OA, C18:1), palmitic acid (PA, C16:0), and stearic acid (C18:0) were obtained from Sigma-Aldrich. The FFA with FFA-free bovine serum albumin (BSA) solution was prepared as described previously. ${ }^{31}$

\section{Nile Red Staining}

After designated treatments, OUMS-29 cells were stained with $1 \mu \mathrm{g} / \mathrm{mL}$ Nile red (Sigma-Aldrich) for 5 minutes at room 
temperature, then fixed by $4 \%$ paraformaldehyde (PFA). ${ }^{32}$ After washing the cells with phosphate-buffered saline (PBS), cellular images were obtained with a laser scanning confocal microscopy with wavelength of $488 \mathrm{~nm}$ for excitation and $>528 \mathrm{~nm}$ for emission for lipid stain.

\section{Analysis of Autophagy}

Initially, to assess the autophagic state in FFA-treated cells, FFA/BSA complex solution was diluted in complete medium composed of Dulbecco's modified Eagle's medium supplemented with $10 \%$ fetal bovine serum. Autophagy was determined by detection of the LC3-II protein with or without bafilomycin A1, a vacuolar $\mathrm{H}^{+}$-ATPase inhibitor (Sigma-Aldrich), by immunoblotting analysis and immunofluorescence staining. Next, to analyze autophagic flux, we used the monomeric red fluorescent protein (mRFP)green fluorescent protein (GFP) tandem fluorescent-tagged LC3 (tf-LC3; Addgene, Cambridge, MA). For the tf-LC3 assay, OUMS-29 cells were transfected with plasmid that expressed tf-LC3 with the use of Effecten Transfection Reagent (Qiagen GmbH, Hilden, Germany) at 24 hours after cell plating. In this assay, FFA/BSA complex solution was added to serum-free Dulbecco's modified Eagle's medium. After the designated treatments, cells were fixed with $4 \%$ PFA in PBS. All of the cellular images were obtained with a laser scanning confocal microscope. For assessment of autophagic flux, colocalization of GFP-LC3 and mRFP-LC3 puncta was measured from triplicate experiments by counting a total of 30 to 40 cells and shown as the percentage of the merged signals from the total number of mRFP signals. In addition, OUMS-29 cells, transfected with plasmids that express GFP-LC3 (a kind gift from Dr. Yoshimori; Osaka University, Osaka, Japan) or mRFPLC3 (Addgene), were treated with FFA/BSA complex solution under serum starvation conditions. After designated treatments, cells were fixed with $4 \%$ PFA in PBS, and then immunofluorescence staining was performed for Lamp1 to assess the late stage of autophagy. Colocalization of Lamp1 and GFP-LC3 or mRFP-LC3 was counted in a total of 30 to 40 cells from triplicate experiments. Colocalization in a twocolor image can be visualized in a two-dimensional histogram, whereby the number of pixels with red and green intensities is plotted as a scattergram with each axis representing the intensity of each color. If there is complete colocalization between channels, the scatter plot should show a diagonal line from the bottom left corner to the top right corner.

\section{Analysis of Lysosomal Function}

To analyze lysosomal acidification, OUMS-29 cells treated with FFA were loaded with $1 \mu \mathrm{mol} / \mathrm{L}$ Lysotracker Red (Molecular Probes, Eugene, OR) for 1 hour under growth conditions, followed by fixation with 4\% PFA in PBS. Subsequently, we performed immunofluorescence staining of Lamp1. Lysotracker is a fluorescent dye for labeling acidic organelles, thus Lysotracker-positive Lamp1 indicates that lysosomal acidification is intact. To analyze cathepsin B activity, we used Magic Red Cathepsin B reagent (Immunochemistry Technologies, LLC, Bloomington, $\mathrm{MN}$ ). After designated treatments, cells were loaded with Magic Red Cathepsin B reagent for 1 hour. It is not until the cell-permeant cresyl violet is cleaved by cathepsin that red fluorescence can be detected by a laser scanning confocal microscopy.

\section{Transmission Electron Microscopy}

Liver tissue pieces were fixed with $2 \%$ glutaraldehyde, postfixed in $1 \%$ osmium tetroxide, dehydrated in a graded ethanol series, and embedded in Quetol 812 (Nisshin EM Co., Ltd., Tokyo, Japan). Ultrathin sections were stained with uranyl acetate and lead citrate and were examined by transmission electron microscopy (JEM-1200-EX; JEOL, Tokyo, Japan).

\section{Immunofluorescence Staining}

We fixed cultured cells in 4\% PFA in PBS for 20 minutes, then permeabilized them in $0.1 \%$ Triton X-100 in PBS or $0.1 \%$ saponin in PBS for 10 minutes or 20 minutes, respectively, followed by incubation with primary antibodies for 1 hour at room temperature. After washing with PBS, cells were incubated with the secondary antibodies for 1 hour at room temperature. In some cases, nuclear staining was performed with $2.5 \mu \mathrm{g} / \mathrm{mL}$ propidium iodide. Images were acquired with a laser scanning confocal microscopy. For double labeling, images were acquired sequentially with separate excitation wavelengths (488 $\mathrm{nm}$ and $543 \mathrm{~nm}$ ), then merged.

\section{Immunoblot Analysis}

We homogenized cells and liver tissues in a lysis buffer composed of $0.187 \mathrm{~mol} / \mathrm{L}$ trishydroxymethylaminomethane$\mathrm{HCl}(\mathrm{pH} 6.8), 10 \%$ sodium dodecyl sulfate, and $5 \mathrm{mmol} / \mathrm{L}$ ethylene diamine tetraacetic acid. Equal amounts of protein were separated by polyacrylamide gel electrophoresis (BioRad Laboratories, Hercules, CA). Proteins were transferred to polyvinylidene fluoride microporous membranes (Millipore Corporation, Billerica, MA). The blots were blocked with $0.2 \%$ skimmed milk in PBS that contained $0.1 \%$ Triton $\mathrm{X}-100$ for 1 hour, and incubated overnight at $4^{\circ} \mathrm{C}$ with primary antibodies. Membranes were washed three times for 10 minutes in PBS with $0.1 \%$ Triton X-100. Subsequently, incubation with appropriate horseradish peroxidase-conjugated secondary antibodies (GE Healthcare, Buckinghamshire, UK) was performed for 1 hour at room temperature, followed by washing three times for 10 minutes in PBS with $0.1 \%$ Triton X-100. The blots were visualized with enhanced chemiluminescence (ECL Plus Western blotting Detection Reagents; GE Healthcare). 

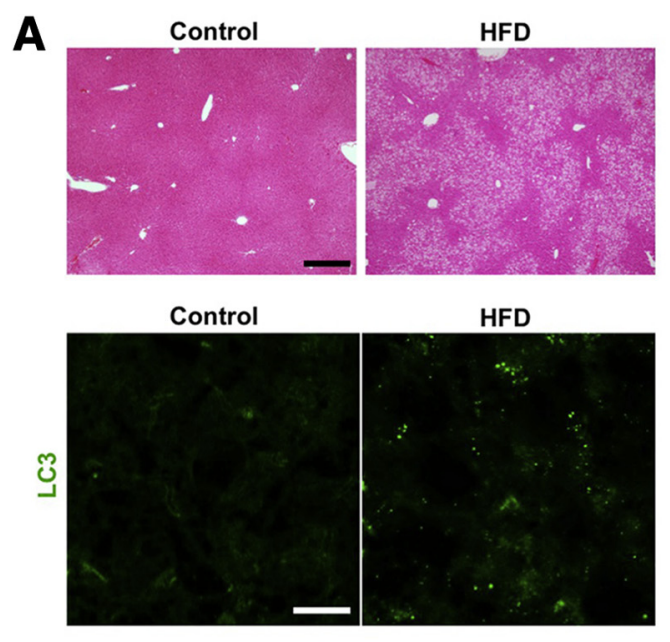

HFD

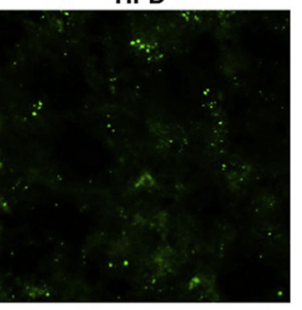

C

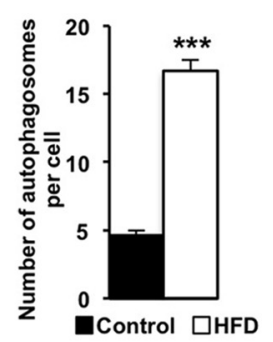

B
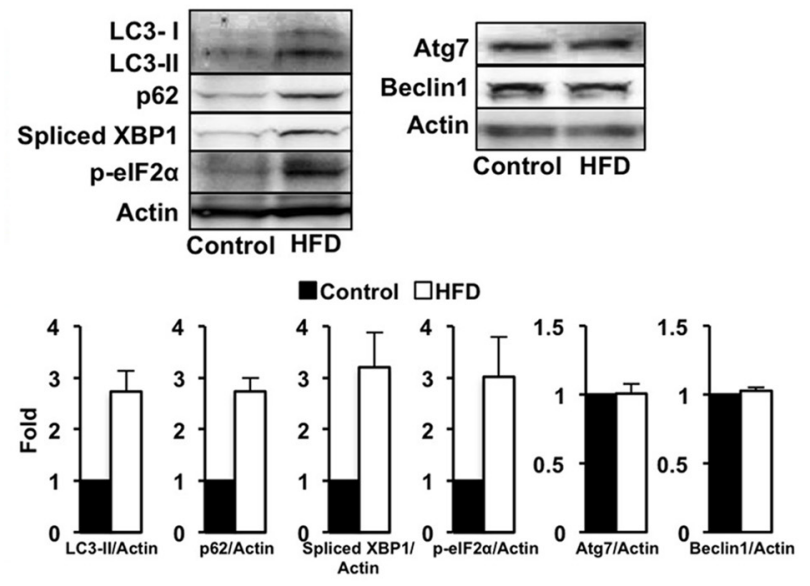

D
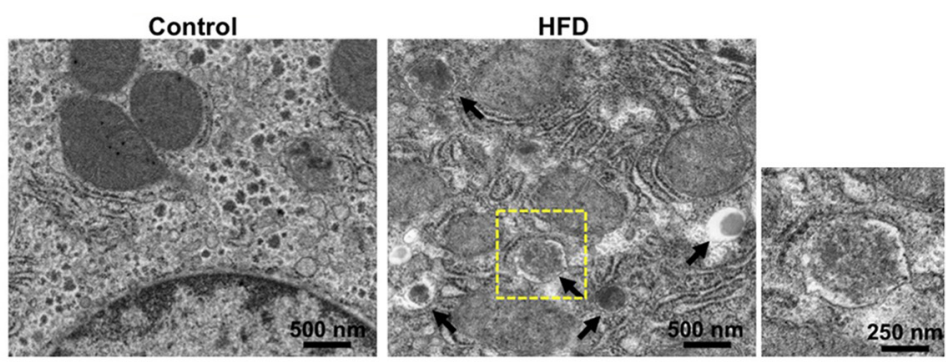

Figure 1 Hepatosteatosis induces the accumulation of autophagosomes coincident with endoplasmic reticulum stress. A: Male C57BL/6J mice were given a control diet or a HFD, as described in the Materials and Methods, followed by hematoxylin and eosin staining. Representative anti-LC3 immunofluorescence staining in liver sections. B: Livers were obtained per condition and subjected to immunoblotting with the indicated antibodies. Densitometry analysis of indicated proteins is shown on the bottom. C: Morphometric analysis of number of autophagosomes per cell. D: Electron micrographs of hepatocytes treated as described in A. Arrows indicate autophagosomes. Boxed area is enlarged in the right panel. Data are presented as means \pm SEM. $n=3$ mice livers for 3 independent experiments (B); $n=30$ different cells of triplicate experiments. ${ }^{* *} P<0.001$, HFD versus control. Scale bars: $500 \mu \mathrm{m}(\mathbf{A}$, top); $10 \mu \mathrm{m}$ (A, bottom). HFD, high-fat diet; LC3, light chain 3; XBP1, X-box binding protein-1.

\section{Statistical Analysis}

Quantitative data (means \pm SEM) were subjected to Student's $t$-test or one-way analysis of variance with post hoc analysis, using SPSS Statistics version 22 (SPSS Inc., Chicago, IL). $P<0.05$ was considered significant.

\section{Results}

\section{Accumulation of Autophagosomes in Obesity}

We initially examined the effect of obesity on autophagy in the mouse liver. For this purpose, male C57BL/6J mice (3 months old) were fed a HFD or a control diet for 12 weeks. At the end of this study, the HFD-fed mice exhibited marked steatosis in hepatocytes compared with the mice fed a control diet (Figure 1A). Next, we analyzed the autophagy of the mice livers by assessing LC3-II protein. The amount of LC3-II is clearly associated with the number of autophagosome membranes and is widely used to monitor autophagy. ${ }^{33}$ We detected LC3-II by immunoblotting and immunostaining. Importantly, because the sensitivity of LC3-II by anti-LC3 antibody tends to be higher than that of
LC3-I, the LC3-II/actin ratio is more appropriate for assessing autophagy level by immunoblotting. ${ }^{34}$ The protein level of LC3-II in the livers of HFD-fed mice was higher than in control mice (Figure 1B). Moreover, we observed a significant increase in LC3-II puncta by immunostaining analysis (Figure 1, A and C). Electron microscopic observation also showed accumulation of autophagosomes concomitant with mildly dilated ER in HFD-fed mice livers compared with control mice (Figure 1D). We measured the expression of p62, which is incorporated into autophagosomes and degraded by lysosomes. ${ }^{35} \mathrm{We}$ observed a greater accumulation of $\mathrm{p} 62$ protein in the livers of HFD-fed mice than in control mice (Figure 1B). A previous report suggested that obesity induced autophagy defect by suppressing the expression of Atg7, which plays a central role in autophagosome formation. ${ }^{16}$ However, no decrease was found in the protein level of Atg7 in the livers of HFD-fed mice in the current study (Figure 1B). We also examined Beclin 1 expression, which is also one of the essential proteins required for the formation of autophagosomes. ${ }^{36}$ No alteration was found in Beclin 1 protein expression in HFD-fed mice livers compared with control mice (Figure 1B). Next, we analyzed ER stress markers, including spliced XBP1 and 
p-eIF2 $\alpha$, in HFD-fed mice livers. Both spliced XBP1 and p-eIF2 $\alpha$ expression increased in HFD-fed mice livers compared with control mice livers (Figure 1B). Collectively, these experiments show that obesity induces the accumulation of autophagosomes coincident with hepatosteatosis and ER stress but does not impair the formation of autophagosomes. To further confirm these results, we performed analyses of autophagy in vitro.

\section{Effects of MUFAs and SFAs on ER Stress and Cellular Steatosis}

OUMS-29 cells were treated with MUFAs, palmitoleic acid or OA, or SFAs, PA or stearic acid, for 12 hours. The cells were stained with Nile red to visualize the lipid droplets. The magnitude of cellular steatosis was greater in cells treated with MUFAs than cells treated with SFAs (Supplemental Figure S1A). For ER stress, SFAs induced a greater elevation of spliced XBP1 and p-eIF2 $\alpha$ proteins than MUFAs in OUMS-29 and Huh7 cells (Supplemental Figure S1B). These results indicate that MUFAs and SFAs have different effects on ER homeostasis and cellular steatosis and that the degree of ER stress is inversely correlated with the magnitude of cellular steatosis.

\section{Impairment of Autophagic Flux in Hepatocytes Treated with SFAs}

We assessed the autophagic level in OUMS-29 cells treated with MUFAs or SFAs. An increase in LC3-II level was observed in cells treated with SFAs, whereas MUFAs had no effect on LC3-II level (Figure 2A). Furthermore, the expression of p62 was elevated in SFA-treated cells but not in MUFA-treated cells, and Atg7 protein levels were unaltered among the treatments with vehicle, MUFAs, and SFAs (Figure 2A). Although a previous study suggested that Beclin 1 could be cleaved by PA-induced caspase activation, ${ }^{37}$ we found that neither MUFA nor SFA treatment induced Beclin 1 cleavage (Figure 2A). Similar results were found in MUFA- or SFA-treated Huh7 cells (Supplemental Figure S2A). We selected OA and PA for the following experiments of autophagic flux because these are the most abundant FAs in the diet and serum. ${ }^{38}$ Furthermore, we used other FFAs in some experiments and obtained similar results with those of OA and PA (data not shown). One of the methods to assess autophagic flux is to monitor the differences in the amount of LC3-II between samples in the presence and absence of lysosomotropic reagents such as bafilomycin A1 or chloroquine, which inhibit lysosomal acidification and autophagosome-lysosome fusion. ${ }^{35,39}$ LC3 turnover assay represents the amount of autophagosomes that is delivered to lysosomes. ${ }^{35} \mathrm{OA}$ treatment alone did not affect LC3-II levels, whereas PA treatment alone induced LC3-II accumulation (Figure 2B). PA but not OA increased the expression levels of p62 in the absence of bafilomycin
A1 (Figure 2B). Whereas concomitant treatment with bafilomycin A1 and OA or PA increased LC3-II and p62 levels, these expression levels were similar compared with bafilomycin A1 alone (Figure 2B). Similar results were found in OA- or PA-treated Huh7 cells (Supplemental Figure S2B). We also evaluated the effect of short-term (6 hours) treatment with FAs in OUMS-29 cells, and similar results were obtained (Supplemental Figure S3). Consistent with these biochemical data, a significant increase in LC3-II-positive autophagosomes was found in cells treated with PA compared with vehicle or OA by immunofluorescence analysis, in the absence of bafilomycin A1 (Figure 2, C and D). In the presence of bafilomycin A1, the number of LC3-II puncta was similar among vehicle, OA and PA (Figure 2, C and D). Moreover, we demonstrated that OA prevented PA-induced ER stress and accumulation of LC3-II and p62, despite accentuating hepatosteatosis (Figure 2, E and F).

\section{Blockage of Late Stage of Autophagy in Hepatocytes Treated with PA}

The current results suggest that SFAs impaired autophagic flux by interruption of the late stage of autophagic pathway, autophagosome-lysosome fusion and subsequent lysosomal degradation. To further elucidate this hypothesis, we used mRFP-GFP tf-LC3 analysis. tf-LC3 assay is a convenient tool to distinguish autophagosomes and autolysosomes. In this assay, autophagosomes show both GFP and mRFP signals, whereas GFP signals are not observed when autophagosome acidification occurs. ${ }^{40}$ OUMS-29 cells transfected with tf-LC3 were incubated with vehicle, OA, or PA under serum starvation conditions. A significant increase in mRFP and GFP overlapping was found in cells treated with PA compared with vehicle or OA, and these signals localized at the perinuclear area (Figure 3). These results indicate that PA impairs autophagosome-lysosome fusion, lysosomal acidification, or lysosomal dysfunction.

\section{PA Inhibits Autophagosome-Lysosome Fusion}

We next compared the location of GFP-LC3 signals to Lamp1, known as a late endosome-lysosome marker, to determine whether PA impaired autophagosome-lysosome fusion. When autophagosomes fuse lysosomes, GFP signals are rapidly attenuated by lysosomal acidic conditions, suggesting that GFP signals and Lamp1 rarely overlap with each other. ${ }^{40}$ To examine the effect of PA on the late stage of autophagy, OUMS-29 cells transfected with GFP-LC3 were incubated with vehicle, OA or PA for 12 hours, under serum starvation conditions, and then cells were stained for Lamp1. PA induced a significant increase in the number of the GFP-LC3 puncta compared with vehicle and OA (Figure 4, A and C). GFP-LC3 rarely colocalized with Lamp1 in cells treated with PA and vehicle or OA (Figure 4, $\mathrm{A}$ and $\mathrm{B})$. Thus, there is a possibility that the impairment 
A

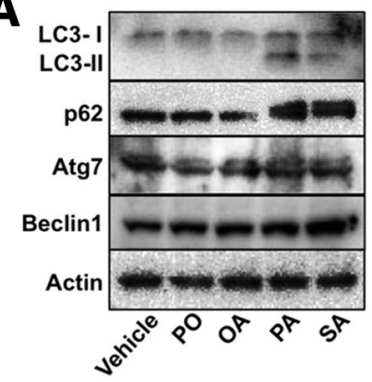

B
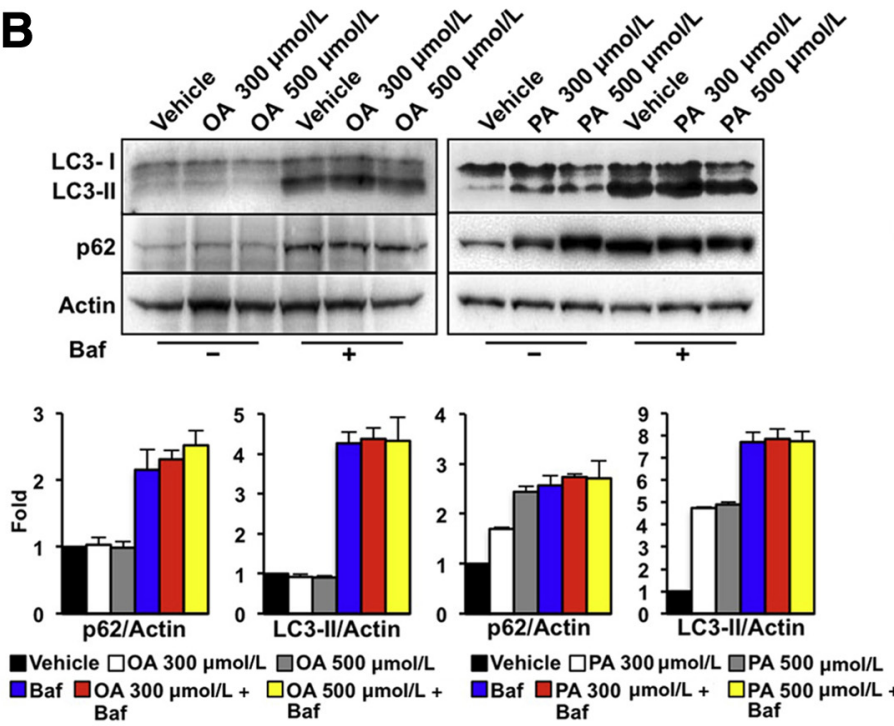

E

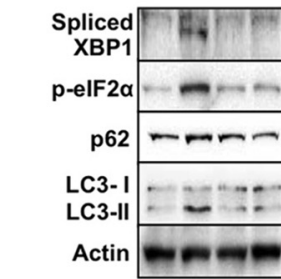

PA $500 \mu \mathrm{mol} / t++$

$\mathrm{OA} 300 \mu \mathrm{mol} / \mathrm{L}--+-$

OA $500 \mu \mathrm{mol} / \mathrm{L}-$ - +
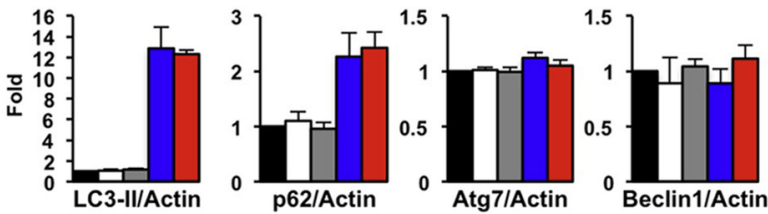

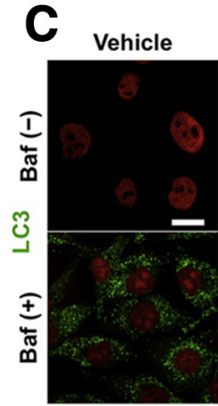

OA

PA

D

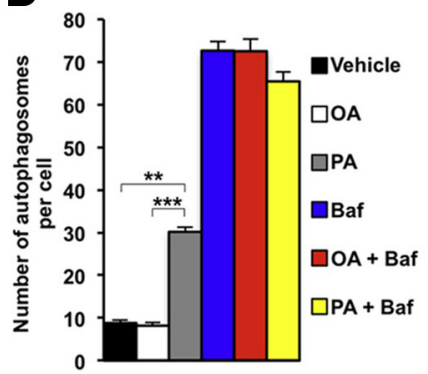

$\mathbf{F}$

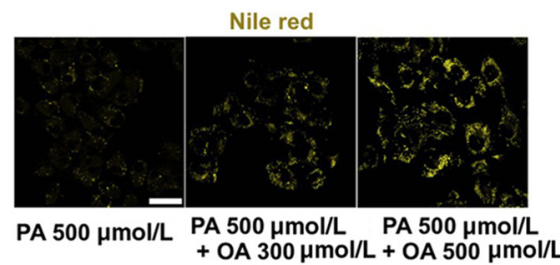

Figure 2 Assessment of autophagic activity in OUMS-29 cells treated with MUFAs or SFAs. FFAs were diluted in complete medium composed of DMEM supplemented with $10 \%$ FBS. A: Immunoblotting analysis of autophagy-related proteins, LC3, p62, Atg7, and Beclin 1, in samples from cells treated with vehicle $(5 \% \mathrm{BSA}), \mathrm{PO}(500 \mu \mathrm{mol} / \mathrm{L}), 0 \mathrm{OA}(500 \mu \mathrm{mol} / \mathrm{L}), \mathrm{PA}(500 \mu \mathrm{mol} / \mathrm{L})$, or SA $(500 \mu \mathrm{mol} / \mathrm{L})$ for 12 hours. Densitometry analysis of indicated proteins is shown on the right. B: LC3 turnover assay. Cells were treated with vehicle $(5 \% \mathrm{BSA}), 0 \mathrm{~A}(500 \mu \mathrm{mol} / \mathrm{L})$, or PA $(500 \mu \mathrm{mol} / \mathrm{L})$ for 12 hours with/without $50 \mathrm{nmol} / \mathrm{L}$ Baf. Representative immunoblotting with the indicated antibodies. Densitometry analysis of indicated proteins is shown on the bottom. C: 0 UMS-29 cells were treated as described in B, followed by immunofluorescence analysis for LC3. D: LC3-II puncta were measured from experiments by counting a total of 30 to 40 OUMS-29 cells. E: 0UMS-29 cells were treated with vehicle $(5 \%$ BSA), PA $(500 \mu \mathrm{mol} / \mathrm{L})$, or PA $(500 \mu \mathrm{mol} / \mathrm{L})$ plus $0 \mathrm{~A}(300 \mu \mathrm{mol} / \mathrm{L})$, or $0 \mathrm{~A}(500 \mu \mathrm{mol} / \mathrm{L})$ for 12 hours. Representative immunoblotting with the indicated antibodies. Densitometry analysis of indicated proteins is shown on the right. F: Nile red staining was performed on OUMS-29 cells treated as described in $\mathbf{E}$. Data are presented as means \pm SEM. $n=3$ independent experiments. ${ }^{* *} P<0.01$, PA versus vehicle; ${ }^{* * *} P<0.001$, PA versus OA. Scale bars: $20 \mu \mathrm{m}(\mathbf{C}) ; 50 \mu \mathrm{m}$ (E). Atg7, autophagy-related 7; Baf, bafilomycin A1; BSA, bovine serum albumin; DMEM, Dulbecco's modified Eagle's medium; FBS, fetal bovine serum; FFA, free fatty acid; LC3, light chain 3; MUFA, monounsaturated fatty acid; OA, oleic acid; PA, palmitic acid; P0, palmitoleic acid; SA, stearic acid; SFA, saturated fatty acid.

of autophagic flux by PA was due to prevention of autophagosome-lysosome fusion. To confirm this hypothesis, we compared the number of mRFP-LC3 signals that colocalized with Lamp1 between vehicle and PA. We found that many mRFP signals colocalized with Lamp1 in the cells treated with vehicle, whereas PA treatment suppressed colocalization of mRFP signals with Lamp1 (Figure 4, $\mathrm{D}-\mathrm{F})$. These results indicate that PA impairs autophagic 


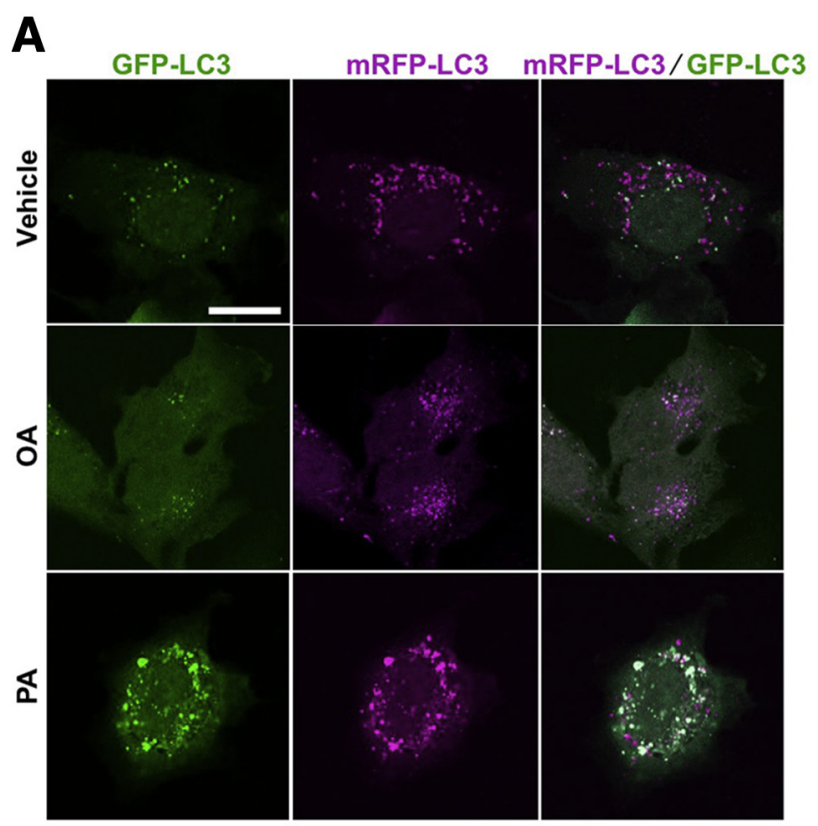

B

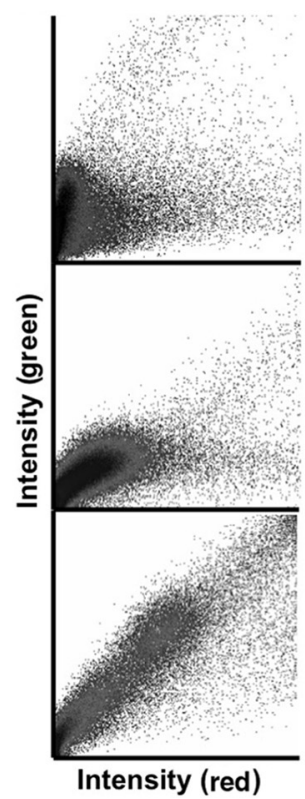

C

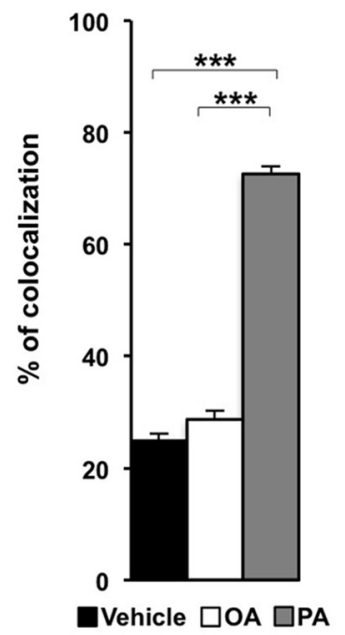

Figure 3 Blockage of late stage of autophagy in cells treated with PA. FFAs were diluted in the serum-free DMEM. A: 0UMS-29 cells, transfected with the mRFP-GFP tandem fluorescent-tagged LC3 plasmids, were treated with vehicle $(5 \% \mathrm{BSA}), 0 \mathrm{~A}(500 \mu \mathrm{mol} / \mathrm{L})$, or PA $(500 \mu \mathrm{mol} / \mathrm{L})$ for 12 hours, followed by fixation and analyzed by microscopy. B: The corresponding two-dimensional histograms for each image in $\mathbf{A}$. The red signals are on the $x$ axis and the green signals are on the $y$ axis in the two-dimensional histogram. C: Colocalization efficiency of mRFP and GFP signals was measured from experiments by counting a total of 30 to 40 OUMS-29 cells, and it was shown as the percentage of the merged signals from the total number of mRFP signals. Data are expressed as means \pm SEM. $n=3$ experiments. ${ }^{* *} P<0.001$, PA versus vehicle or OA. Scale bar $=20 \mu \mathrm{m}$. BSA, bovine serum albumin; DMEM, Dulbecco's modified Eagle's medium; FFA, free fatty acid; GFP, green fluorescent protein; LC3, light chain 3; mRFP, monomeric red fluorescent protein; OA, oleic acid; PA, palmitic acid.

flux by preventing the fusion of autophagosomes and lysosomes.

\section{PA-Induced Impairment of Autophagic Flux Is Not Due to Lysosomal Dysfunction}

We next examined the lysosomal acidification in OUMS29 cells after treatment with PA by using Lysotracker Red, which is a fluorescent dye that accumulates in acidic compartments. ${ }^{41}$ OUMS-29 cells treated with PA were labeled for Lysotracker Red and then stained with Lamp1. We could not identify Lysotracker ${ }^{-}$Lamp1 in PA-treated cells and vehicle, suggesting that lysosomal acidification was not impaired by PA treatment (Figure 5A). Lysotracker-positive Lamp1 was not identified in bafilomycin A1-treated cells (Figure 5A). Next we monitored the levels of cathepsin B enzyme activity with the use of Magic Red cathepsin B detection kit. The cell-permeant cresyl violet is cleaved if cathepsin enzymes are active, and this produces red fluorescent signals that can be detected. Thus, the red fluorescence intensity correlates with cathepsin B activity. ${ }^{42}$ The red fluorescence intensity in PA-treated cells was comparable with vehicle, whereas bafilomycin A1 reduced the fluorescence intensity (Figure 5B). These results suggest that neither acidification of lysosomes nor the activity of lysosomal proteases is impaired in PA-treated cells.

\section{Induction of Selective Autophagic Sequestration of Aggregated Proteins in HFD-Fed Mice Livers and PA-Treated Cells}

Immunofluorescence analysis confirmed that ubiquitin and p62-positive inclusions, Mallory-Denk bodies, ${ }^{43}$ were observed in the HFD-fed mice livers (Figure 6A) and PAtreated cultured cells (Supplemental Figure S4). However, they could not be detected in the livers of control mice (Figure 6A) and OA-treated cultured cells (Supplemental Figure S4). We examined whether selective autophagic sequestration of aggregated proteins was induced in the livers of HFD-fed mice and PA-treated cells. The level of ubiquitinated proteins increased in the HFD-fed mice livers compared with control mice livers (Figure 6B). In addition, the phosphorylation of p62 at Ser351, which is considered to be indispensable for selective autophagy of aggregated proteins, ${ }^{44}$ increased only in the HFD-fed mice livers (Figure 6B). In addition, in the livers of HFD-fed mice, LC3-positive autophagosomes occasionally colocalized with ubiquitin-positive inclusions (Figure 6C). In cultured cells, PA but not OA increased the expression levels of phosphorylation of p62 at Ser351 and ubiquitinated proteins (Figure 6D). Moreover, PA-induced accumulation of Ser351-phosphorylated p62 and ubiquitinated proteins further increased on bafilomycin A1 administration, whereas this finding was not observed in OA-treated cells (Figure 6D). 


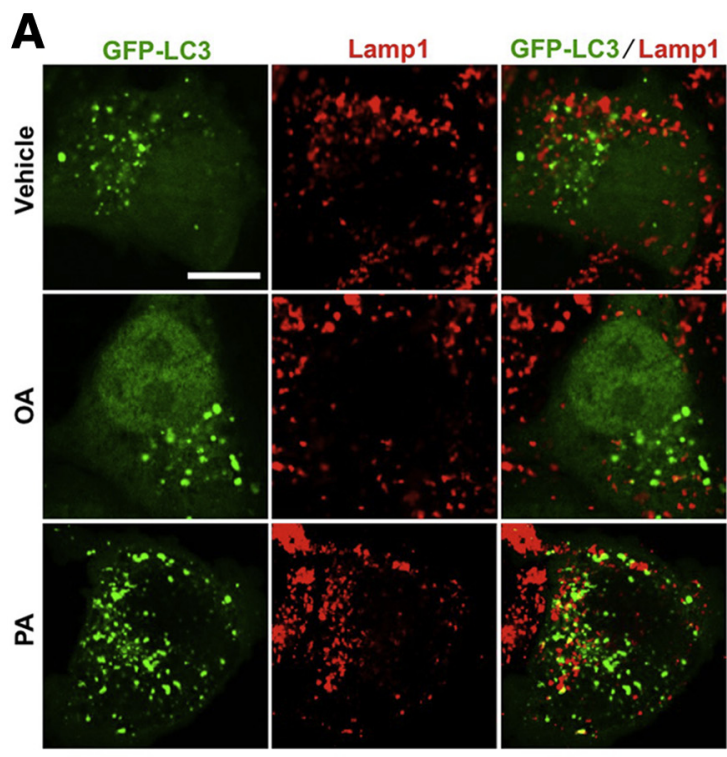

D mRFP-LC3

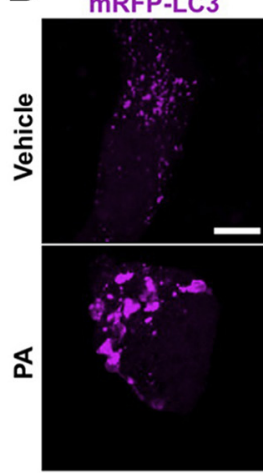

Lamp1

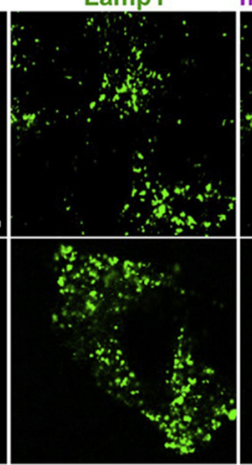

mRFP-LC3/Lamp

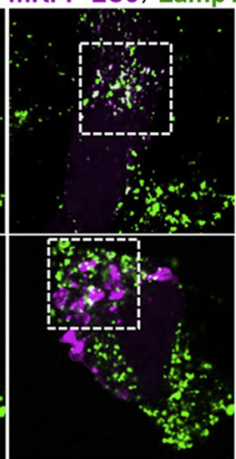

B

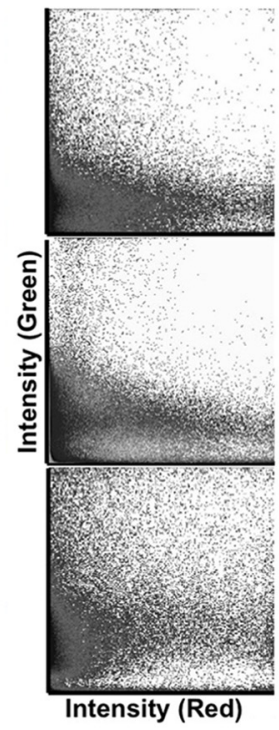

E
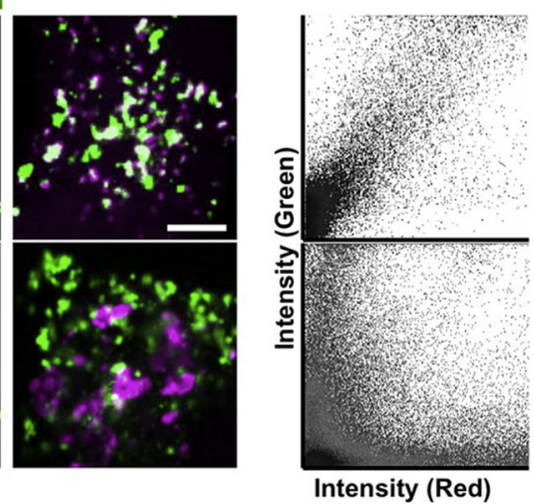

C

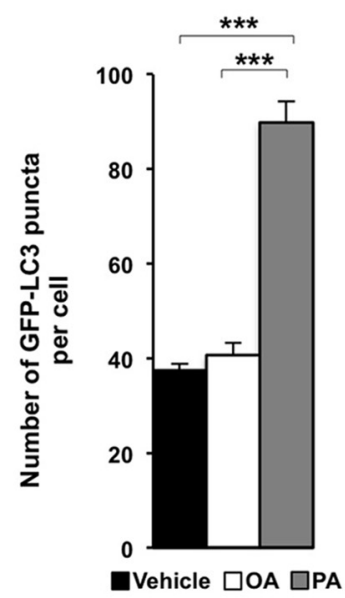

$\mathbf{F}$

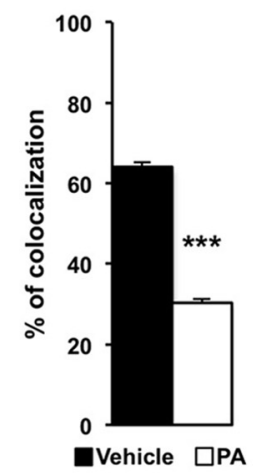

Figure 4 PA impairs autophagosome-lysosome fusion. A: OUMS-29 cells, transfected with the GFP-LC3 plasmids, were treated with vehicle (5\% BSA), 0A $(500 \mu \mathrm{mol} / \mathrm{L})$, or PA $(500 \mu \mathrm{mol} / \mathrm{L})$ for 12 hours in the absence of serum, followed by fixation, and immunofluorescence staining for Lamp1 was performed. B: The corresponding two-dimensional histograms for each image in A. The red signals are on the $x$ axis, and the green signals are on the $y$ axis in the twodimensional histogram. C: The number of GFP-LC3 puncta per cell was quantified from triplicate experiments by counting a total of 30 to 40 OUMS-29 cells. D: OUMS-29 cells, transfected with the mRFP-LC3 plasmids, were treated with vehicle ( $5 \% \mathrm{BSA})$ or PA $(500 \mu \mathrm{mol} / \mathrm{L})$ as described in $\mathbf{A}$, followed by fixation, and immunofluorescence staining for Lamp1 was performed. Boxed areas are enlarged in the right panels. E: The corresponding two-dimensional histograms for each image in $\mathbf{D}$. The red signals are on the $x$ axis, and the green signals are on the $y$ axis in the two-dimensional histogram. F: Colocalization efficiency of mRFP and Lamp1 was measured from experiments by counting a total of 30 to 40 OUMS-29 cells and is shown as the percentage of the merged signals from the total number of mRFP signals. Data are expressed as means \pm SEM. $n=3$ experiments. ${ }^{*} * P<0.001$, PA versus vehicle or 0 A. Scale bars: $10 \mu \mathrm{m}(\mathbf{A}$ and $\mathbf{D}) ; 5$ $\mu \mathrm{m}$ (D, right panels). BSA, bovine serum albumin; FFA, free fatty acid; GFP, green fluorescent protein; Lamp1, lysosomal-associated membrane protein 1; LC3, light chain 3; $m$ RFP, monomeric red fluorescent protein; OA, oleic acid; PA, palmitic acid.

These phenomenons imply that selective autophagic sequestration of aggregated proteins is induced in PA- but not OAtreated cells. Indeed, immunofluorescence analysis revealed that the inclusions composed of ubiquitin colocalized with GFP-labeled autophagosomes in PA-treated cells (Figure 6E).

\section{PA-Induced Blockage of Autophagic Flux Is Associated with the ER Stress}

To clarify whether ER homeostasis was involved in the PA-induced impairment of autophagic flux, we evaluated the effect of chemical chaperon on PA-induced disruption of autophagic flux. 4-Phenylbutyric acid, one of the chemical chaperones, ${ }^{45}$ reduced the PA-induced elevation of spliced XBP1, p-eIF2 $\alpha$, LC3-II, and p62 in OUMS-29 cells (Figure 7A). In the presence of bafilomycin A1, the restorative effect on autophagic flux by 4-phenylbutyric acid was abrogated, and the protein level of LC3-II was equivalent between PA and PA + 4-phenylbutyric acid (Figure 7A). However, puromycin, which induces ER stress by inhibition of protein synthesis, ${ }^{46}$ exacerbated PAinduced LC3-II accumulation in parallel with the elevation of ER stress markers, although puromycin alone did not alter autophagic flux (Figure 7B). In the presence of 

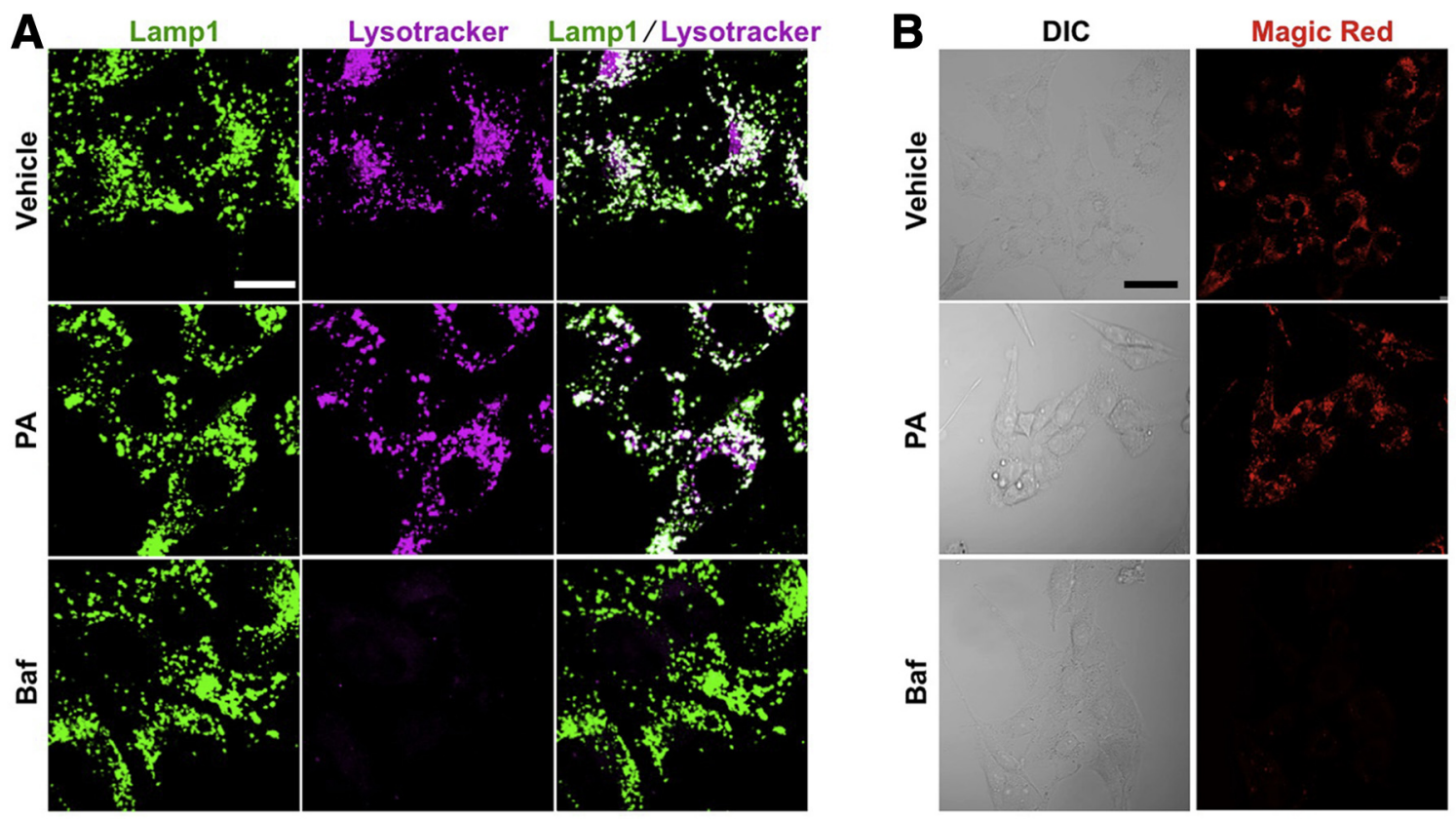

Figure 5 Assessment of lysosomal acidification and cathepsin B activity in PA-treated cells. A: 0UMS-29 cells treated with vehicle (5\% BSA), PA $(500 \mu \mathrm{mol} / \mathrm{L})$, or Baf $(50 \mathrm{nmol} / \mathrm{L})$ for 12 hours in the absence of serum were loaded with $1 \mu \mathrm{mol} / \mathrm{L}$ Lysotracker Red for 1 hour, followed by fixation, and immunofluorescence staining for Lamp1 was performed. No Lysotracker-negative Lamp1 was found in PA-treated cells and vehicle (5\% BSA). B: 0UMS-29 cells were treated as described in A. Cells were then loaded with Magic Red Cathepsin B reagent for 1 hour and then analyzed by microscopy. DIC images are shown on the left, and fluorescence images are shown on the right. Scale bars: $20 \mu \mathrm{m}$ (A); $50 \mu \mathrm{m}$ (B). Baf, bafilomycin A1; BSA, bovine serum albumin; DIC, differential interferential contrast; Lamp1, lysosomal-associated membrane protein 1; PA, palmitic acid.

bafilomycin A1, the protein level of LC3-II was equivalent between PA and PA + puromycin (Figure 7B). Consistent with these results, tf-LC3 fluorescence analysis showed that the colocalization efficiency of mRFP-LC3 with GFP-LC3 correlated with ER stress (Figure 7, C and D). These results suggest that the disruption of ER homeostasis is involved in the PA-induced blockage of autophagic flux.

\section{Discussion}

In obesity, hepatic steatosis decreases the level of hepatic autophagy because of several molecular mechanisms. ${ }^{16,27,28}$ In this study, lipid overload induced accumulation of autophagosomes in parallel with ER stress. Moreover, we demonstrated that the accumulation of autophagosomes was due to the impairment of autophagosome-lysosome fusion without change in lysosomal-degradation function and that this impairment of autophagic flux was associated with ER stress.

We first showed that a HFD induced accumulation of autophagosomes as manifested by increased LC3-II levels (Figure 1, A-D). In addition, we also confirmed that a larger amount of the protein p62 was accumulated in the livers of HFD-fed mice than in control mice livers (Figure 1B). Importantly, an increase in autophagosomes indicates not only enhancement of autophagy but also blockade of late stage of autophagy. ${ }^{35}$ Moreover, we should be careful when assessing the significance of p62 expression. Although the accumulation of p62 was thought to indicate a decrease in autophagic degradation, p62 levels are also enhanced by other factors such as stress due to proteasome inhibition. ${ }^{13}$ Recent investigation found that p62 is restored during prolonged starvation due to transcriptional up-regulation of $\mathrm{p} 62$ and de novo protein synthesis. ${ }^{47}$ These studies suggest that the expression level of p62 does not always inversely correlate with autophagic activity. Then, to assess the autophagic flux in more detail, we performed analyses of autophagic flux in cultured hepatocytes exposed to FFAs. In our study, ER stress was observed in cultured hepatocytes treated with SFAs, and this coincided with the accumulation of LC3-II and p62 (Supplemental Figure S1 and Figure 2A). The same was observed in the livers of HFD-fed mice (Figure 1B). However, MUFAs did not increase the expression levels of the ER stress markers, LC3-II, and p62 (Supplemental Figure S1 and Figure 2A). These findings suggest that it is reasonable to use cultured hepatocytes loaded with SFAs as a substitute for HFD-fed mice to assess hepatic autophagy in obesity. Furthermore, in PA- but not OA-treated cells, ubiquitin and p62-positive inclusions were identified (Supplemental Figure S4). This finding also supports that PA-treated cells can be used as an in vitro model to evaluate autophagy in nonalcoholic steatohepatitis, because ubiquitin and p62positive inclusions, Mallory-Denk bodies, are known as a marker of nonalcoholic steatohepatitis pathologic process. ${ }^{43,48}$

In cultured hepatocytes, LC3 turnover assay and monitoring tf-LC3 signals indicated that OA does not alter 


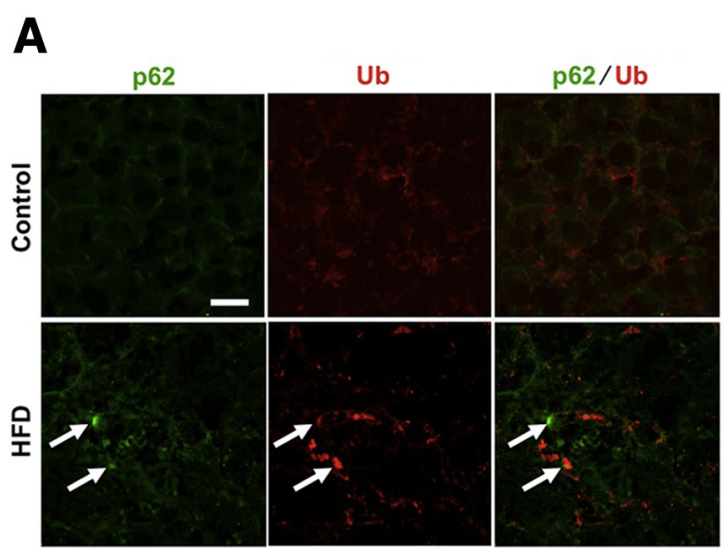

C
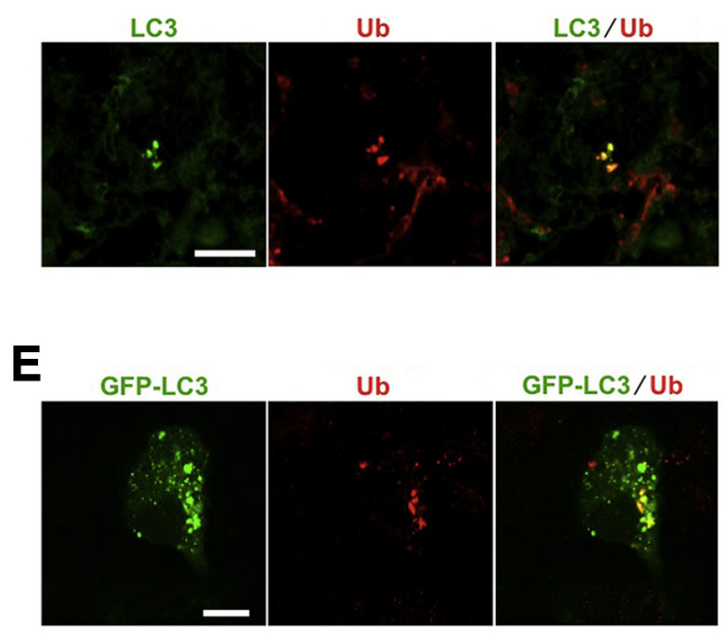
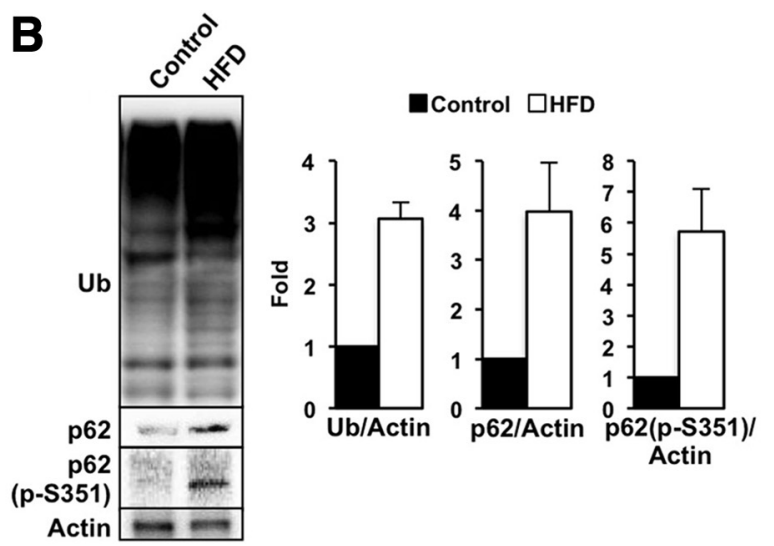

D
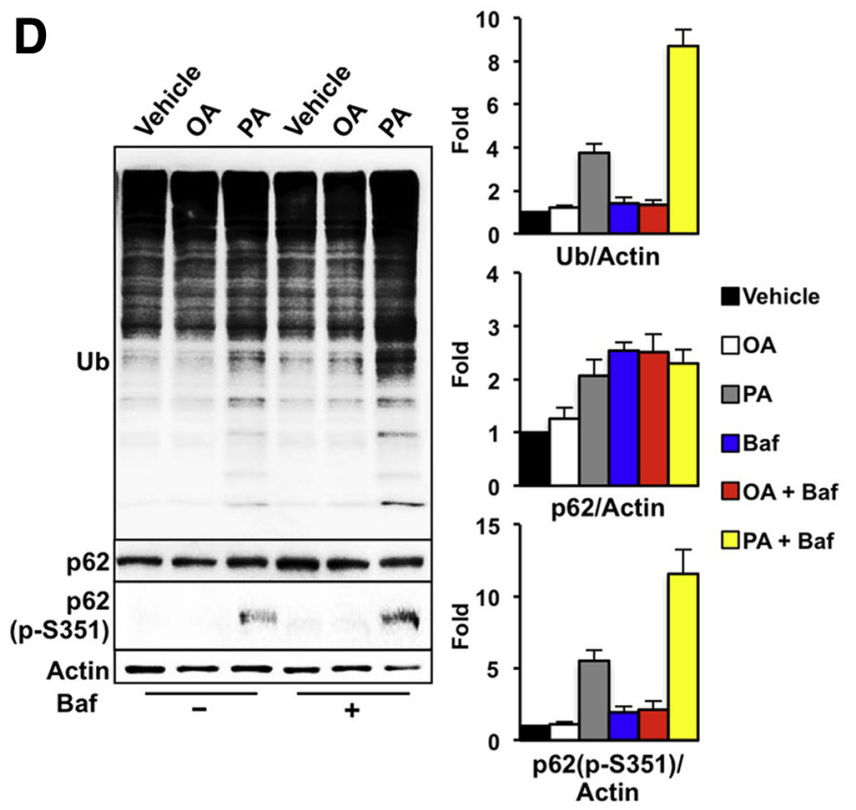

Figure 6 Induction of selective autophagy of aggregated proteins in HFD-fed mice livers and PA-treated cells. A: Livers from mice given a control diet or a HFD were double-immunostained with p62 and ubiquitin antibodies. Arrows indicate inclusions. B: Livers were obtained per condition and subjected to immunoblotting with the indicated antibodies. Densitometry analysis of indicated proteins is shown on the right. C: Livers from mice given a control diet or a HFD were double-immunostained with LC3 and ubiquitin antibodies. D: FFAs were diluted in the serum-free DMEM. 0UMS-29 cells were treated with vehicle (5\% BSA), OA $(500 \mu \mathrm{mol} / \mathrm{L})$, or PA $(500 \mu \mathrm{mol} / \mathrm{L})$ for 12 hours with/without $50 \mathrm{nmol} / \mathrm{L}$ Baf. Representative immunoblotting with the indicated antibodies. Densitometry analysis of indicated proteins is shown on the right. E: OUMS-29 cells, transfected with the GFP-LC3 plasmid, were treated with $500 \mu \mathrm{mol} / \mathrm{L}$ PA for 12 hours, followed by fixation, and immunofluorescence staining for ubiquitin was performed. Data are expressed as means \pm SEM. $n=3$ independent experiments (B and D); $n=3$ livers per condition (B). Scale bars: $20 \mu \mathrm{m}$ (A and E); $10 \mu \mathrm{m}$ (C). Baf, bafilomycin A1; BSA, bovine serum albumin; DMEM, Dulbecco's modified Eagle's medium; FFA, free fatty acid; GFP, green fluorescent protein; HFD, high-fat diet; LC3, light chain 3; OA, oleic acid; PA, palmitic acid; Ub, ubiquitin.

hepatic autophagic flux, whereas PA suppresses degradation of contents in the autophagosomes (Figure 2, B-D, and Figure 3). A previous study found that a defect in lysosomal acidification and proteinase activity of cathepsin impaired hepatic autophagic degradation in obesity. ${ }^{27}$ However, our results indicated that PA-induced impairment of autophagic flux was due to blockage of autophagosome-lysosome fusion, without alteration in lysosomal environment, including acidification and hydrolytic function (Figures 4 and 5). Although autophagy has been considered as a random cytoplasmic degradation system, recent reports suggest that selective autophagy is induced in response to cellular stress. ${ }^{22,23}$ In our study, selective autophagic sequestration of aggregated proteins is induced in HFD-fed mice livers and PA-treated cells (Figure 6, C and E), suggesting that, although lipotoxicity interferes with the autophagosome-lysosome fusion, selective autophagy is induced to remove harmful proteins.

ER stress was shown to be a strong inducer of autophagy. ${ }^{49}$ However, a previous report showed that thapsigargin, which triggers ER stress by depletion of luminal calcium store, blocks fusion of autophagosomes with lysosomes, resulting in accumulation of autophagosomes without change in autophagosome formation. ${ }^{50}$ These indicate that ER stress does not necessarily activate autophagy. In our study, in fact, PA did not stimulate the step of autophagosome formation despite the increase in ER stress. On the contrary, PA-induced ER stress contributed to the impairment 

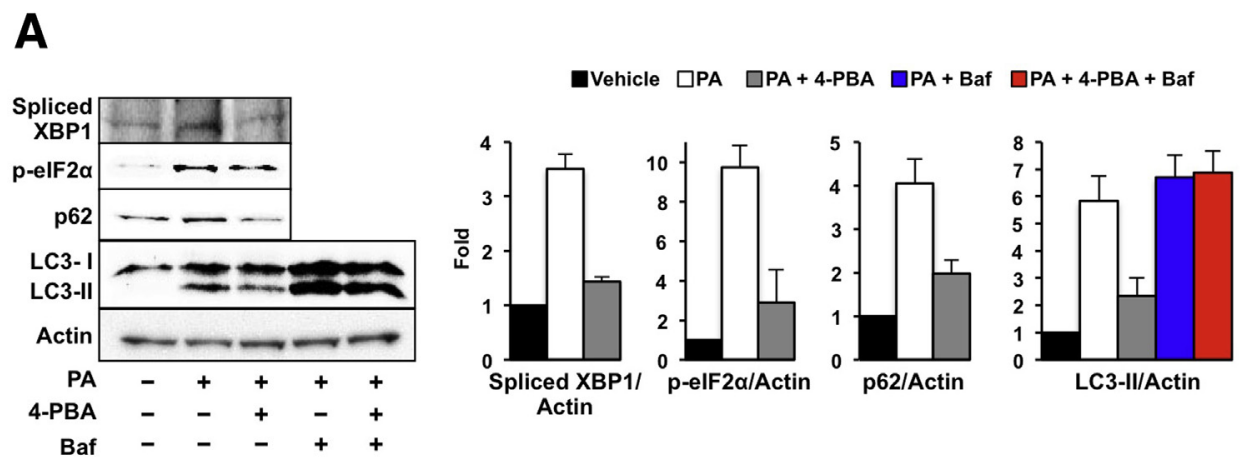

\section{B}

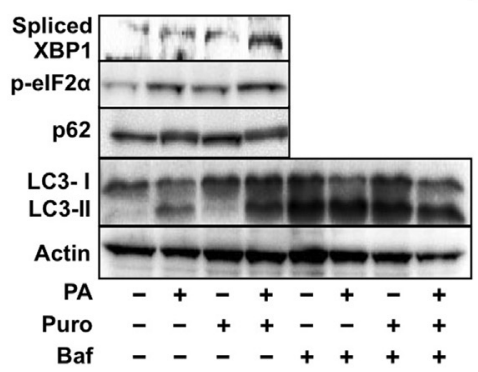

$\square$ Vehicle $\square$ PA $\square$ Puro $\square \mathrm{PA}+$ Puro $\square$ Baf $\square \mathrm{PA}+$ Baf $\square$ Puro + Baf $\square \mathrm{PA}+$ Puro + Baf

\section{C}

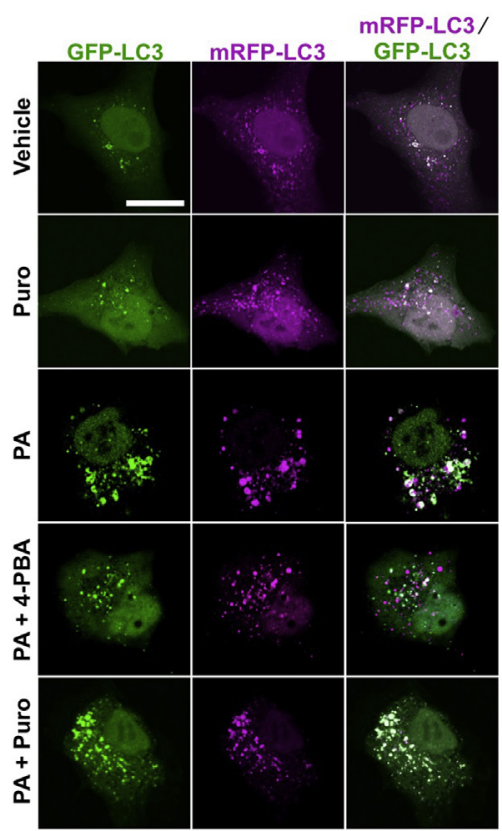

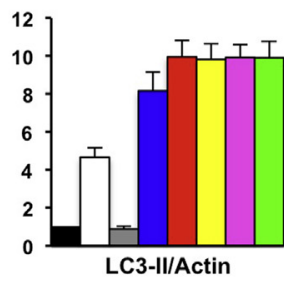

Actin

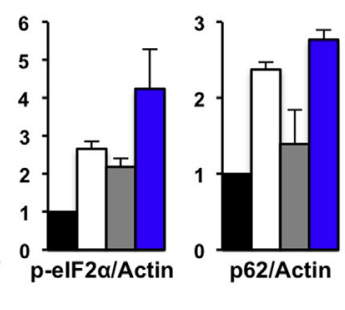

D

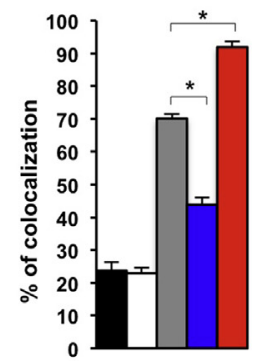

Vehicle

$\square$ Puro

$\square \mathrm{PA}$

$\square \mathrm{PA}+4-\mathrm{PBA}$

$\square P A+$ Puro

Figure 7 The effect of 4-PBA and puromycin on ER stress and autophagic flux in OUMS-29 cells treated with PA. A: 0UMS-29 cells were pretreated in the absence or presence of $2 \mathrm{mmol} / \mathrm{L}$ 4-PBA for 2 hours and then treated with $500 \mu \mathrm{mol} / \mathrm{L}$ PA with/without $50 \mathrm{nmol} / \mathrm{L}$ Baf for 6 hours. Representative immunoblotting with the indicated antibodies. Densitometry analysis of indicated proteins is shown on the right. B: 0UMS- 29 cells were pretreated in the absence or presence of $50 \mu \mathrm{g} / \mathrm{mL}$ Puro for 6 hours and then treated with $500 \mu \mathrm{mol} / \mathrm{L}$ PA with/without $50 \mathrm{nmol} / \mathrm{L}$ Baf for 6 hours. Representative immunoblotting with the indicated antibodies. Densitometry analysis of indicated proteins is shown on the right. C: OUMS-29 cells, transfected with tf-LC3 plasmids, were treated with vehicle $(5 \% \mathrm{BSA})$, Puro $(50 \mu \mathrm{g} / \mathrm{mL})$, PA $(500 \mu \mathrm{mol} / \mathrm{L})$, PA $(500 \mu \mathrm{mol} / \mathrm{L})+4-\mathrm{PBA}(2 \mathrm{mmol} / \mathrm{L})$, or PA $(500 \mu \mathrm{mol} / \mathrm{L})+$ puromycin $(50 \mu \mathrm{g} / \mathrm{mL})$ as described in $\mathbf{A}$ and $\mathbf{B}$, followed by fixation and analyzed by microscopy. D: Colocalization efficiency of mRFP and GFP signals was measured from triplicate experiments by counting a total of 50 to 60 OUMS-29 cells, and it is shown as the percentage of the merged signals from the total number of mRFP signals. Data are expressed as means \pm SEM. $n=3$ independent experiments. ${ }^{*} P<0.05$, PA versus PA +4 -PBA or PA + Puro. Scale bar $=20 \mu \mathrm{m} .4-\mathrm{PBA}, 4$-phenylbutyric acid; Baf, bafilomycin A1; BSA, bovine serum albumin; ER, endoplasmic reticulum; GFP, green fluorescent protein; LC3, light chain 3; mRFP, monomeric red fluorescent protein; PA, palmitic acid; $\mathrm{p}$-eIF2a, phosphorylation of $\alpha$ subunit of eukaryotic initiation factor 2; Puro, puromycin; tf-LC3, tandem fluorescent-tagged light chain 3; XBP1, X-box binding protein-1. 
of autophagosome-lysosome fusion (Figure 7). Consistent with our study, others also demonstrated that the chemical chaperone suppresses the increase in LC3-II and accumulation of autophagosomes induced by thapsigargin in parallel with reduction of ER stress. ${ }^{51}$ Thus, we speculate that the disturbance of ER homeostasis caused by obesity alters autophagic flux. Moreover, we found in this study that OA prevented PA-induced impairment of autophagic flux, despite accentuating hepatosteatosis (Figure 2, E and F). These findings collectively indicate the possibility that the inhibitory effect of lipid overload on autophagic flux is associated with the disruption of ER homeostasis rather than the intracellular accumulation of lipid droplets. However, further studies are required to identify this possibility.

\section{Conclusions}

The impairment of autophagosome-lysosome fusion is the cause of accumulation of autophagosomes observed in the HFD-fed mice livers and in cultured hepatocytes treated with SFAs. In these models, however, selective autophagy of ubiquitin-positive aggregated proteins is induced to remove harmful proteins. Moreover, the impairment of autophagic flux in SFA-treated cells is ameliorated by the reduction of ER stress. Although autophagy has been better known as a protective mechanism against disease progression in obesity-linked complications, ${ }^{16}$ overactivation of autophagy can also be harmful in some conditions. ${ }^{52}$ Our results may point the way to a new therapeutic strategy for restoration of selective autophagy in nonalcoholic steatohepatitis and prevention of subsequent severe conditions such as cirrhosis and hepatocellular carcinoma.

\section{Acknowledgments}

Lamp1 antibody was a kind gift from J. Thomas August (Johns Hopkins University). OUMS-29 cells, transfected with plasmids expressing GFP-LC3, were a kind gift from Dr. Tamotsu Yoshimori (Osaka University).

\section{Supplemental Data}

Supplemental material for this article can be found at http://dx.doi.org/10.1016/j.ajpath.2016.03.003.

\section{References}

1. Bechmann LP, Hannivoort RA, Gerken G, Hotamisligil GS, Trauner M, Canbay A: The interaction of hepatic lipid and glucose metabolism in liver diseases. J Hepatol 2012, 56:952-964

2. Ortiz-Lopez C, Lomonaco R, Orsak B, Finch J, Chang Z, Kochunov VG, Hardies J, Cusi K: Prevalence of prediabetes and diabetes and metabolic profile of patients with nonalcoholic fatty liver disease (NAFLD). Diabetes Care 2012, 35:873-878

3. Loomba R, Abraham M, Unalp A, Wilson L, Lavine J, Doo E, Bass NM; Nonalcoholic Steatohepatitis Clinical Research Network:
Association between diabetes, family history of diabetes, and risk of nonalcoholic steatohepatitis and fibrosis. Hepatology 2012, 56: 943-951

4. Paredes AH, Torres DM, Harrison SA: Nonalcoholic fatty liver disease. Clin Liver Dis 2012, 16:397-419

5. Milić S, Stimac D: Nonalcoholic fatty liver disease/steatohepatitis: epidemiology, pathogenesis, clinical presentation and treatment. Dig Dis 2012, 30:158-162

6. Fabbrini E, Sullivan S, Klein S: Obesity and nonalcoholic fatty liver disease: biochemical, metabolic, and clinical implications. Hepatology 2010, 51:679-689

7. Gregor MF, Hotamisligil GS: Thematic review series: adipocyte biology. Adipocyte stress: the endoplasmic reticulum and metabolic disease. J Lipid Res 2007, 48:1905-1914

8. Dara L, Ji C, Kaplowitz N: The contribution of endoplasmic reticulum stress to liver diseases. Hepatology 2011, 53:1752-1763

9. Malhi H, Kaufman RJ: Endoplasmic reticulum stress in liver disease. J Hepatol 2011, 54:795-809

10. Ron D, Walter P: Signal integration in the endoplasmic reticulum unfolded protein response. Nat Rev Mol Cell Biol 2007, 8 $519-529$

11. McCracken AA, Brodsky JL: Assembly of ER-associated protein degradation in vitro: dependence on cytosol, calnexin, and ATP. J Cell Biol 1996, 132:291-298

12. Hamel FG: Preliminary report: inhibition of cellular proteasome activity by free fatty acids. Metabolism 2009, 58:1047-1049

13. Harada M, Hanada S, Toivola DM, Ghori N, Omary MB: Autophagy activation by rapamycin eliminates mouse Mallory-Denk bodies an blocks their proteasome inhibitor-mediated formation. Hepatology 2008, 47:2026-2035

14. Kroeger H, Miranda E, MacLeod I, Pérez J, Crowther DC, Marciniak SJ, Lomas DA: Endoplasmic reticulum-associated degradation (ERAD) and autophagy cooperate to degrade polymerogenic mutant serpins. J Biol Chem 2009, 284:22793-22802

15. Tremblay F, Krebs M, Dombrowski L, Brehm A, Bernroider E, Roth E, Nowotny P, Waldhäusl W, Marette A, Roden M: Overactivation of S6 kinase 1 as a cause of human insulin resistance during increased amino acid availability. Diabetes 2005, 54: 2674-2684

16. Yang L, Li P, Fu S, Calay ES, Hotamisligil GS: Defective hepatic autophagy in obesity promotes ER stress and causes insulin resistance. Cell Metab 2010, 11:467-478

17. Rautou PE, Mansouri A, Lebrec D, Durand F, Valla D, Moreau R: Autophagy in liver diseases. J Hepatol 2010, 53: $1123-1134$

18. Otoda $\mathrm{T}$, Takamura $\mathrm{T}$, Misu $\mathrm{H}$, Ota $\mathrm{T}$, Murata $\mathrm{S}$, Hayashi $\mathrm{H}$, Takayama H, Kikuchi A, Kanamori T, Shima KR, Lan F, Takeda T, Kurita S, Ishikura $\mathrm{K}$, Kita $\mathrm{Y}$, Iwayama $\mathrm{K}$, Kato $\mathrm{K}$, Uno $\mathrm{M}$, Takeshita Y, Yamamoto M, Tokuyama K, Iseki S, Tanaka K, Kaneko S: Proteasome dysfunction mediates obesity-induced endoplasmic reticulum stress and insulin resistance in the liver. Diabetes 2013, 62:811-824

19. Mehrpour M, Esclatine A, Beau I, Codogno P: Autophagy in health and disease. 1. Regulation and significance of autophagy: an overview. Am J Physiol Cell Physiol 2010, 298:C776-C785

20. Orenstein SJ, Cuervo AM: Chaperone-mediated autophagy: molecular mechanisms and physiological relevance. Semin Cell Dev Biol 2010, 21:719-726

21. Mizushima N, Komatsu M: Autophagy: renovation of cells and tissues. Cell 2011, 147:728-741

22. Nixon RA: The role of autophagy in neurodegenerative disease. Nat Med 2013, 19:983-997

23. Komatsu M: Liver autophagy: physiology and pathology. J Biochem $2012,152: 5-15$

24. Hara T, Nakamura K, Matsui M, Yamamoto A, Nakahara Y, Suzuki-Migishima R, Yokoyama M, Mishima K, Saito I, Okano H, Mizushima N: Suppression of basal autophagy in neural cells 
causes neurodegenerative disease in mice. Nature 2006, 441: 885-889

25. Singh R, Kaushik S, Wang Y, Xiang Y, Novak I, Komatsu M, Tanaka K, Cuervo AM, Czaja MJ: Autophagy regulates lipid metabolism. Nature 2009, 458:1131-1135

26. Harada M, Strnad P, Toivola DM, Omary MB: Autophagy modulates keratin-containing inclusion formation and apoptosis in cell culture in a context-dependent fashion. Exp Cell Res 2008, 314: $1753-1764$

27. Inami $Y$, Yamashina $S$, Izumi $K$, Ueno $T$, Tanida I, Ikejima $K$, Watanabe S: Hepatic steatosis inhibits autophagic proteolysis via impairment of autophagosomal acidification and cathepsin expression. Biochem Biophys Res Commun 2011, 412:618-625

28. Koga H, Kaushik S, Cuervo AM: Altered lipid content inhibits autophagic vesicular fusion. FASEB J 2010, 24:3052-3065

29. Malhi H, Bronk SF, Werneburg NW, Gores GJ: Free fatty acids induce JNK-dependent hepatocyte lipoapoptosis. J Biol Chem 2006, 281:12093-12101

30. Akazawa Y, Cazanave S, Mott JL, Elmi N, Bronk SF, Kohno S, Chariton MR, Gores GJ: Palmitoleate attenuates palmitate-induced Bim and PUMA up-regulation and hepatocyte lipoapoptosis. J Hepatol 2010, 52:586-593

31. Cousin SP, Hügl SR, Wrede CE, Kajio H, Myers MG Jr, Rhodes CJ: Free fatty acid-induced inhibition of glucose and insulin-like growth factor I-induced deoxyribonucleic acid synthesis in the pancreatic beta-cell line INS-1. Endocrinology 2001, 142:229-240

32. Greenspan P, Mayer EP, Fowler SD: Nile red: a selective fluorescent stain for intracellular lipid droplets. J Cell Biol 1985, 100: 965-973

33. Kabeya Y, Mizushima N, Ueno T, Yamamoto A, Kirisako T, Noda T, Kominami E, Ohsumi Y, Yoshimori T: LC3, a mammalian homologue of yeast Apg8p, is localized in ahtophagosome membranes after processing. EMBO J 2000, 19:5720-5728

34. Mizushima N, Yoshimori T: How to interpret LC3 immunoblotting. Autophagy 2007, 3:542-545

35. Mizushima N, Yoshimori T, Levine B: Methods in mammalian autophagy research. Cell 2010, 140:313-326

36. He C, Levine B: The Beclin 1 interactome. Curr Opin Cell Biol 2010, 22:140-149

37. Mei S, Ni HM, Manley S, Bockus A, Kassel KM, Luyendyk JP, Copple BL, Ding WX: Differential roles of unsaturated and saturated fatty acid on autophagy and apoptosis in hepatocytes. J Pharmacol Exp Ther 2011, 339:487-498

38. Baylin A, Kabagambe EK, Siles X, Campos H: Adipose tissue biomarkers of fatty acid intake. Am J Clin Nutr 2002, 76:750-757

39. Rubinsztein DC, Cuervo AM, Ravikumar B, Sarkar S, Korolchuk V, Kaushik S, Koionsky DJ: In search of an "autophagomometer". Autophagy 2009, 5:585-589
40. Kimura S, Noda T, Yoshimori T: Dissection of the autophagosome maturation process by a novel reporter protein, tandem fluorescenttagged LC3. Autophagy 2007, 3:452-460

41. Bampton ET, Goemans CG, Niranjan D, Mizushima N, Tolkovsky AM: The dynamics of autophagy visualized in live cells: from autophagosome formation to fusion with endo/lysosomes. Autophagy 2005, 1:23-36

42. Ni HM, Bockus A, Wozniak AL, Jones K, Weinman S, Yin XM, Ding WX: Dissecting the dynamic turnover of GFP-LC3 in the autolysosome. Autophagy 2011, 7:188-204

43. Zatloukal K, French SW, Stumptner C, Strnad P, Harada M, Toivola DM, Cadrin M, Omary MB: From Mallory to Mallory-Denk bodies: what, how and why? Exp Cell Res 2007, 313:2033-2049

44. Taguchi K, Fujikawa N, Komatsu M, Ishii T, Unno M, Akaike T, Motohashi H, Yamamoto M: Keap1 degradation by autophagy for the maintenance of redox homeostasis. Proc Natl Acad Sci U S A 2012, 109:13561-13566

45. Hanada S, Harada M, Kumemura H, Bishr Omary M, Koga H, Kawaguchi T, Taniguchi E, Yoshida T, Hisamoto T, Yanagimoto C, Maeyama M, Ueno T, Sata M: Oxidative stress induces the endoplasmic reticulum stress and facilitates inclusion formation in cultured cells. J Hepatol 2007, 47:93-102

46. Yanagitani $\mathrm{K}$, Imagawa $\mathrm{Y}$, Iwawaki $\mathrm{T}$, Hosoda A, Saito M, Kimata Y, Kohno K: Cotranslational targeting of XBP1 protein to the membrane promotes cytoplasmic splicing of its own mRNA. Mol Cell 2009, 34:191-200

47. Sahani MH, Itakura E, Mizushima N: Expression of the autophagy substrate SQSTM1/p62 is restored during prolonged starvation depending on transcriptional upregulation and autophagy-derived amino acids. Autophagy 2014, 10:431-441

48. Zatloukal K, Stumptner C, Fuchsbichler A, Heid H, Schnoelzer M, Kenner L, Kleinert R, Prinz M, Aguzzi A, Denk H: p62 is a common component of cytoplasmic inclusions in protein aggregation diseases. Am J Pathol 2002, 160:255-263

49. Ogata M, Hino S, Saito A, Morikawa K, Kondo S, Kanemoto S, Murakami T, Taniguchi M, Tanii I, Yoshinaga K, Shiosaka S, Hammarback JA, Urano F, Imaizumi K: Autophagy is activated for cell survival after endoplasmic reticulum stress. Mol Cell Biol 2006, 26:9220-9231

50. Ganley IG, Wong PM, Gammoh N, Jiang X: Distinct autophagosomallysosomal fusion mechanism revealed by thapsigargin-induced autophagy arrest. Mol Cell 2011, 42:731-743

51. Kim DS, Li B, Rhew KY, Oh HW, Lim HD, Lee W, Chae HJ, Kim HR: The regulatory mechanism of 4-phenylbutyric acid against ER stress-induced autophagy in human gingival fibroblasts. Arch Pharm Res 2012, 35:1269-1278

52. Yang Z, Klionsky DJ: Eaten alive: a history of macroautophagy. Nat Cell Biol 2010, 12:814-822 